\title{
Reggeized Gluons with a Running Coupling Constant
}

\author{
M. A. Braun \\ Department of high-energy physics, \\ University of S. Petersburg, 198904 S. Petersburg, Russia and \\ Department of Particle Physics, University of Santiago de Compostela, \\ 15706 Santiago de Compostela, Spain
}

July 1994

\begin{abstract}
The equation for two reggeized gluons in the vacuum channel is generalized to take into account the running QCD coupling constant on the basis of the bootstrap condition for gluon reggeization. Both the gluon trajectory as a function of momentum and the interaction as a function of distance grow like $\log \log$ in the ultraviolet. The resulting equation depends on the confinement region. With a simple parametrization of its influence by an effective gluon mass the pomeron intercept turns out much smaller than for a fixed coupling constant (the BFKL pomeron).
\end{abstract}

\section{US-FT/12-94}




\section{Introduction}

Recently much effort has been spent generalizing the BFKL pomeron [ 1 ] to include the running QCD coupling constant [2 ]. As in the first attempts by Lipatov et al. [3] the idea is to introduce the running constant directly into the equation for the vacuum channel on the basis of more or less plausible assumptions about the momentum or distance scale at which the gluons interact. Such an approach ignores the origin of the BFKL equation, which is, in fact, a direct consequence of the assumption that the gluon reggeizes. This assumption can be formalized in the so-called bootstrap condition [ 3$]$ : in the gluon channel the two-gluon equation should possess a solution in the form of the gluon Regge pole. One can then verify that with the BFKL interaction kernel and the known gluon Regge-trajectory this condition is indeed satisfied.

The bootstrap condition is the basic assumption in deriving the BFKL pomeron, which may be considered as its byproduct in relation to the vacuum channel. For that reason, in our opinion, all attempts to change the BFKL equation in order to introduce the running coupling constant should start with the bootstrap equation rather than with the vacuum channel one. One has to change simultaneously both the gluon interaction and its trajectory in a manner consistent with the bootstrap requirement. The present note is devoted to this problem.

We first prove that the bootstrap equation admits more general solutions than the known BFKL one. These involve a generalized interaction kernel, parametrized by a function of a single momentum variable, related to the gluon Regge trajectory via a nonlinear integral equation. We determine both the kernel and the trajectory asymptotically from the known behaviour of the gluon density with the running coupling constant. As a result the trajectory turns out rising like $\ln \ln q^{2}$ at high momenta rather than like $\ln q^{2}$ as with a fixed coupling constant. The behaviour of both the kernel and the trajectory at low momenta $q^{2} \leq \Lambda^{2}$ remains undetermined and governed by the confinement. However the bootstrap equation guarantees that all the good properties of the interaction kernel for reggeized gluons remain the same as for the fixed coupling constant BFKL case: the kernel results insensitive to the infrared region. This leaves some hope that the results will depend only weakly on the details of the confinement mechanism.

Parametrizing the confinement effects by a single parameter (the "gluon mass" $m$ ) and interpolating both the trajectory and the kernel between the ultraviolet and infrared regions by their asymptotic expressions at high momenta appropriately modified to match the low momentum behaviour we investigate the resulting equation for the pomeron at $q=0$. Contrary to the fixed constant case the pomeron singularity now becomes a pole in the $j$ plane whose position depends on the single dimensionless parameter $m / \Lambda \geq 1$. In the limit $m \rightarrow \Lambda$ the equation becomes singular and effectively coincides with the BFKL one. With growing $m$, as variational calculations indicate, the pole moves to the left so that the intercept lies much closer to unity than for the BFKL pomeron. According to these variational estimates, the pomeron intercept (minus one) $\Delta$ with the running coupling constant for reasonable values of $m / \Lambda$ weakly depends on this ratio and lies in the region of $0.15-0.17$.

\section{The bootstrap equation and its solution}

The bootstrap condition is a result of the requirement that the equation for two reggeized gluons have a Regge pole solution in the gluon channel. Let $\alpha(q)=1+\omega(q)$ be the gluon Regge trajectory and $K\left(q, q_{1}, q_{1}^{\prime}\right)$ be the two-gluon interaction kernel. The (homogeneous) 
equation for two reggeized gluons in colour representation $R$ is then

$$
\left(j-1-\omega\left(q_{1}\right)-\omega\left(q_{2}\right)\right) \psi\left(q_{1}\right)=\lambda_{R} \int\left(d^{2} q_{1}^{\prime} /(2 \pi)^{2}\right) K\left(q, q_{1}, q_{1}^{\prime}\right) \psi\left(q_{1}^{\prime}\right)
$$

where $q=q_{1}+q_{2}$ is the total momentum, $j$ is the angular momentum and $\lambda_{R}$ is the strength of the interaction for a given colour representation. For colour group $S U(3)$ in the gluon channel $\lambda_{8}=3 / 2$ and in the vacuum channel $\lambda_{1}=2 \lambda_{8}=3$. Concentrating on the gluon channel and requiring that the solution of (1) be of the form of a Regge pole with a trajectory $1+\omega(q)$, one arrives at the bootstrap equation

$$
\left(\omega(q)-\omega\left(q_{1}\right)-\omega\left(q_{2}\right)\right) \psi\left(q_{1}\right)=\lambda_{8} \int\left(d^{2} q_{1}^{\prime} /(2 \pi)^{2}\right) K\left(q, q_{1}, q_{1}^{\prime}\right) \psi\left(q_{1}^{\prime}\right)
$$

It is a complicated functional-integral equation which relates $\omega, K$ and $\psi$. Following the known result for the BFKL pomeron we shall seek solutions of (2) with a constant $\psi\left(q_{1}\right)$. Then the bootstrap equation simplifies to a relation between $\omega$ and $K$ :

$$
\omega(q)-\omega\left(q_{1}\right)-\omega\left(q_{2}\right)=\lambda_{8} \int\left(d^{2} q_{1}^{\prime} /(2 \pi)^{2}\right) K\left(q, q_{1}, q_{1}^{\prime}\right)
$$

It is easy to find that this relation can be satisfied provided $K$ is chosen in the form

$$
K\left(q, q_{1}, q_{1}^{\prime}\right)=\left(\eta\left(q_{1}\right) / \eta\left(q_{1}^{\prime}\right)+\eta\left(q_{2}\right) / \eta\left(q_{2}^{\prime}\right)\right) / \eta\left(q_{1}-q_{1}^{\prime}\right)-\eta(q) / \eta\left(q_{1}^{\prime}\right) \eta\left(q_{2}^{\prime}\right)
$$

where the function $\eta(q)$ is related to the trajectory $\omega(q)$ by a nonlinear integral equation

$$
\omega(q)=-\lambda_{8} \eta(q) \int\left(d^{2} q_{1} /(2 \pi)^{2}\right) / \eta\left(q_{1}\right) \eta\left(q_{2}\right)
$$

Equations (4) and (5) allow to find the kernel $K$ for a given gluon trajectory $\omega(q)$ or vice versa. The BFKL pomeron results from a particular solution of (4) and (5) with

$$
\eta(q)=2 \pi q^{2} / g^{2}
$$

where $g$ is the fixed coupling constant.

The form (4) garantees that if $\eta(q) \rightarrow 0$ as $q \rightarrow 0$ the kernel $K$ remains nonsingular at $q_{1}^{\prime}=q_{2}^{\prime}=0$ and goes to zero as $q_{1}, q_{2} \rightarrow 0$. One can observe that the leading contributions of the two terms in (4) cancel in these limits. One can also prove that, with (4) and (5) satisfied, the equation for two gluons in the vacuum channel is nonsingular at $q_{1}-q_{1}^{\prime} \rightarrow 0$. To do that use the trick of [ 3 ] and represent the trajectory (5) as

$$
\omega(q)=-2 \lambda_{8} \eta(q) \int\left(d^{2} q_{1} /(2 \pi)^{2}\right) / \eta\left(q_{1}\right)\left(\eta\left(q_{1}\right)+\eta\left(q_{2}\right)\right)
$$

Then, remembering that $\lambda_{1}=2 \lambda_{8}$, in the vacuum channel Eq. (1) can be written as

$$
\begin{gathered}
(j-1) \psi\left(q_{1}\right)=\lambda_{1} \int\left(d^{2} q_{1}^{\prime} /(2 \pi)^{2}\right)\left(( \eta ( q _ { 1 } ) / \eta ( q _ { 1 } - q _ { 1 } ^ { \prime } ) ) \left(\psi\left(q_{1}^{\prime}\right) / \eta\left(q_{1}^{\prime}\right)-\psi\left(q_{1}\right) /\left(\eta\left(q_{1}^{\prime}\right)+\eta\left(q_{1}-q_{1}^{\prime}\right)\right)\right.\right. \\
\left.+(1 \leftrightarrow 2)-\eta(q) \psi\left(q_{1}^{\prime}\right) / \eta\left(q_{1}^{\prime}\right) \eta\left(q_{2}^{\prime}\right)\right)
\end{gathered}
$$

With $\eta(0)=0$ the singularity at $q_{1}=q_{1}^{\prime}$ on the right-hand side is evidently cancelled between the terms coming from the kernel $K$ and the trajectory $\omega$. This cancellation has been long known for the BFKL pomeron. 


\section{The running coupling constant}

According to the considerations of the preceding section to generalize for the running coupling constant one has to find with it either the Regge trajectory $\omega(q)$ and then construct the kernel $K$ using (4) and (5) or directly the kernel $K$ with the structure (4), that is, the function $\eta(q)$, and then find the trajectory from (5). We shall use the latter approach. To determine $\eta(q)$ we consider the vacuum channel equation (8) at $q=0$, which is equivalent to studying the gluon distribution for virtuality $q_{1}^{2}=q_{2}^{2}$ :

$$
\begin{gathered}
(j-1) \psi\left(q_{1}\right)=2 \lambda_{1} \int\left(d^{2} q_{1}^{\prime} /(2 \pi)^{2}\right)\left(( \eta ( q _ { 1 } ) / \eta ( q _ { 1 } - q _ { 1 } ^ { \prime } ) ) \left(\psi\left(q_{1}^{\prime}\right) / \eta\left(q_{1}^{\prime}\right)\right.\right. \\
\left.-\psi\left(q_{1}\right) /\left(\eta\left(q_{1}^{\prime}\right)+\eta\left(q_{1}-q_{1}^{\prime}\right)\right)-\eta(q) \psi\left(q_{1}^{\prime}\right) / \eta\left(q_{1}^{\prime}\right)^{2}\right)
\end{gathered}
$$

Let $q_{1} \rightarrow \infty$. Then the dominant (logarithmic) contribution comes only from the first term, from the integration region $q_{1}^{\prime}<q_{1}$. In this limit the equation becomes

$$
(j-1) \psi\left(q_{1}\right)=2 \lambda_{1} \int\left(d^{2} q_{1}^{\prime} /(2 \pi)^{2}\right) \theta\left(q_{1}^{2}-q_{1}^{\prime 2}\right) \psi\left(q_{1}^{\prime}\right) / \eta\left(q_{1}^{\prime}\right)
$$

Comparing this with the well-known behavior of the gluon distribution at large $q_{1}^{2}$ we find asymptotically

$$
1 / \eta(q)=g^{2}(q) / 2 \pi q^{2}=2 \pi / b q^{2} \ln q^{2} / \Lambda^{2}
$$

where $g(q)$ is the QCD running coupling constant,

$$
b=\left(11-(2 / 3) N_{F}\right) / 4
$$

and $\Lambda \simeq 200 M e v$ is the standard QCD parameter. Relation (11) is valid for $q^{2}>>\Lambda^{2}$. So the comparison to the usual evolution equation allows to determine $\eta(q)$ for large values of the argument.

The equation for two reggeized gluons (1) involves all values of $q^{2}$, smaller than $\Lambda^{2}$ included. As we have observed in Sec. 2, the equation is stable respective to possible singularities of $1 / \eta(q)$ as $q \rightarrow 0$. However it does not mean that the equation does not depend on small values of $q^{2}$ at all. At most we may expect that this dependence is weak. Thus we are faced with the problem of prolongation of the asymptotical expression for $\eta(q)$ (and the kernel $K$ ) into the region of $q^{2}$ comparable to $\Lambda^{2}$ and even smaller than $\Lambda^{2}$. This cannot be done on the basis of any theoretical considerations. The region of small $q^{2}$ is that of confinement where, in fact, the equation for two gluons itself looses sense. Rather we have to invoke some initial values for the function $\eta(q)$ at small $q^{2}$ which effectively take into account the influence of confinement.

The asymptotical expression (11) blows up at $q^{2}=\Lambda^{2}$ (the infrared pole). A simple way to avoid it is to introduce "the gluon mass" $m$ changing $q^{2}$ to $q^{2}+m^{2}$. Thus we assume for all $q^{2}$

$$
1 / \eta(q)=2 \pi / b\left(q^{2}+m^{2}\right) \ln \left(\left(q^{2}+m^{2}\right) / \Lambda^{2}\right)
$$

In this formula $\Lambda$ is the observable QCD parameter and $m$ is a new parameter which incorporates the confinement effects. Evidently to avoid the infrared pole we have to choose $m>\Lambda$. The limiting case is when $m=\Lambda$ and the pole appears at $q=0$. As mentioned due to the properties of the kernel the vacuum channel equation remains valid for this especially simple case with no more parameters than $\Lambda$. As calculation show, values $q^{2}<<\Lambda^{2}$ are essential in the vacuum equation with $m=\Lambda$. Inspection of (13) then shows that this limit is equivalent to the BFKL pomeron with a coupling constant $g^{2}=2 \pi / b$. One should keep in mind, however, than there are no a priori theoretical arguments that might support the choice $m=\Lambda$ and that the values $q^{2}<<\Lambda^{2}$ essential in this limit are completely unphysical (deep inside the confinement region). Thus the BFKL pomeron appears as a formal but unphysical limit of the equation with a running coupling constant with $\eta\left(q_{1}\right)$ given by (13). 


\section{The pomeron at $q=0$}

At $q=0$ the equation (1) can be rewritten in a simpler form changing the function $\psi$ according to

$$
\psi(q) \rightarrow \eta(q) \psi(q)
$$

Introduce the two-gluon potential $V$ with a kernel in the momentum space

$$
V(q)=1 / \eta(q)
$$

Then we obtain an equation

$$
(1-2 \eta(q)) \psi(q)=2 \lambda_{1} \int\left(d^{2} q^{\prime} /(2 \pi)^{2}\right) V\left(q-q^{\prime}\right) \psi\left(q^{\prime}\right)-\lambda_{1} \eta(0) V(q) \int\left(d^{2} q^{\prime} /(2 \pi)^{2}\right) V\left(q^{\prime}\right) \psi\left(q^{\prime}\right)
$$

It has the form of the stationary Schroedinger equation in two dimensions

$$
H \psi=E \psi
$$

where the energy is $E=1-j$ and the Hamiltonian is

$$
H=-2 \omega(q)-2 \lambda_{1} V+\lambda_{1} \eta(0)|V><V|
$$

The rightmost singularity in $j$ corresponds to the ground state energy of the Hamiltonian (18).

In the Hamiltonian (18) the Regge trajectory $\omega(q)$ plays the role of the kinetic energy. The interaction consists of two parts. The first one is a normal local pair interaction with a potential

$$
V(r)=\int\left(d^{2} q /(2 \pi)^{2}\right) V(q) \exp i q r
$$

The second part (the last term in (18)) is a separable interaction, which appears in our case because according to (13) $\eta(0) \neq 0$ unless $m=\Lambda$. With $\eta(q)$ given by (13) both $\omega(q)$ and $V$ are completely determined by (5) and (15). However their explicit expressions in terms of momentum space integrals are rather complicated and can be evaluated only by numerical calculations. To have some idea about the behaviour of $\omega(q)$ and $V(r)$ we therefore start by studying their asymptotical expressions for small and large values of their corresponding arguments.

In the following we assume $\Lambda=1$.

Let us begin with the Regge trajectory. Its explicit expression is given by (5) and (13):

$$
\omega(q)=-\left(\lambda_{8} / 2 \pi b\right)\left(q^{2}+m^{2}\right) \ln \left(q^{2}+m^{2}\right) \int d^{2} q_{1} /\left(q_{1}^{2}+m^{2}\right) \ln \left(q_{1}^{2}+m^{2}\right)\left(q_{2}^{2}+m^{2}\right) \ln \left(q_{2}^{2}+m^{2}\right)
$$

with $q_{1}+q_{2}=q$. Evidently $\omega(q)<0$ for all $q$. At $q=0$ it is finite:

$$
\omega(0)=-\left(\lambda_{8} / 2 b\right)\left(1+m^{2} \ln m^{2} \operatorname{E} i\left(-\ln m^{2}\right)\right)
$$

Note that at $m=1$ the second term in (21) is zero and $\omega(0)=-\lambda_{8} / 2 b$. The asymptotical behaviour at $q^{2}>>1$ is provided by the integration over $q_{1}^{2}<<q_{2}$ or $q_{2}^{2}<<q^{2}$ in (20). To study it the form (7) is more convenient. Simple calculations give the asymptotical expression

$$
\omega(q)_{q^{2}>>1} \simeq-\left(\lambda_{8} / b\right) \ln \left(\ln q^{2} / \ln m^{2}\right)
$$

where we have retained the $\ln \ln m^{2}$ term to show the divergence at $m=1$. As in the BFKL case this divergence is cancelled by the contribution from the interaction in Eq. (1). 
The potential $V(r)$ is explicitly given by

$$
V(r)=(1 / 2 \pi b) \int d^{2} q \exp i q r /\left(q^{2}+m^{2}\right) \ln \left(q^{2}+m^{2}\right)
$$

It goes to zero as $r \rightarrow \infty$

$$
V(r=\infty)=0
$$

The asymptotical behaviour at small $r$ is determined by the behavior of $\eta(q)$ at large $q^{2}$. Calculations give

$$
V(r)_{r<<1} \simeq(1 / 2 b) \ln \left(\ln \left(1 / r^{2}\right) / \ln m^{2}\right)
$$

As mentioned, in Eq.(1) the terms proportional to $\ln \ln m^{2}$ from (22) and (25) cancel due to the relation $\lambda_{1}=2 \lambda_{8}$.

As one observes, the Hamiltonian (18) possesses quite normal features unless $m=1$. The kinetic energy $-2 \omega(q)$ results positive and steadily grows with $q^{2}$ from its value $E_{0}$ at $q=0$ equal to $-2 \omega(0)$ and given by $(21)$, which only leads to a constant energy shift. The potential energy $-2 \lambda_{1} V(r)$ is negative which means attraction between the gluons, partially compensated by the repulsive separable term. All interaction goes to zero at large values of $r$. At $r \rightarrow 0$ the potential goes to minus infinity, although the singularity at $r=0$ is quite weak. With such an Hamiltonian we expect normal bound states at negative values of the shifted energy $\tilde{E}=E-E_{0}$ which means poles in the $j$-plane.

The obtained asymptotic expressions for $\omega(q)$ and $V(r)$ are of course valid only for large values of $q$ and $1 / r$ respectively. The exact form of both kinetic and interaction energy has to be calculated numerically from (20) and (23) for a given $m$. We know, however, that on the one side, the equation (1) is not very sensitive to infrared behaviour of $\omega(q)$ and $V(r)$ and, on the other hand, the initial function $\eta(q)$ can only be well determined at $q^{2}>>1$, so that only the asymptotic expressions for $\omega(q)$ at $q^{2}>>1$ and $V(r)$ at $1 / r^{2}>>1$ are well established. For these reasons, instead of using the exact values of $\omega(q)$ and $V(r)$ following from (20) and (23), we shall use their asymptotical expressions (22) and (25) for all $q$ and $r$ respectively, appropriately modifying them to take into account the known values of $\omega(0)$ and $V(r=\infty)$. Namely we shall use Eq. (17) with $E$ changed to $E-E_{0}$ and with the expressions

$$
\begin{gathered}
\omega(q)=-\left(\lambda_{8} / b\right) \ln \left(\ln \left(q^{2}+m^{2}\right) / \ln m^{2}\right) \\
V(r)=(1 / 2 b) \ln \left(\ln \left(1 / r^{2}+m^{2}\right) / \ln m^{2}\right)
\end{gathered}
$$

which agree with $(22)$ and $(25)$ and go to zero at $q=0$ and $1 / r=0$, respectively. The constant term $\ln \ln m^{2}$ is in fact cancelled between the first and second term in (18). In the limit $m=1$ the last (separable) term in (18) disappears and the Hamiltonian takes a simple form

$$
H=\left(2 \lambda_{8} / b\right)\left(\ln \ln \left(1+q^{2}\right)-\ln \ln \left(1+1 / r^{2}\right)\right)
$$

As mentioned, in this limit small values of $q$ and $1 / r$ turn out to be essential. Then the Hamiltonian further simplifies to

$$
H=\left(2 \lambda_{8} / b\right)\left(\ln q^{2}+\ln r^{2}\right)
$$

which is nothing but the BFKL Hamiltonian up to an energy shift.

\section{Variational calculations of the pomeron intercept}

For all its nice look, Eq. (17) with kinetic and interaction terms given by (26) and (27) is much more difficult to solve than the BFKL one due to the lack of scale invariance. So 
instead of trying to solve it exactly we investigate the ground state energy by the standard variational technique. Evidently the lowest energy eigenvalue $E=1-j$ depends only on the dimensionless ratio of the gluon mass $m$ to the QCD parameter $\Lambda=1$.

We have taken our trial functions as linear combinations of the two-dimensional harmonic oscillator functions for zero angular momentum

$$
\psi\left(r^{2}\right)=\sum_{k=0}^{N} c_{k} \mathrm{~L}_{k}\left(a r^{2}\right) \exp \left(-a r^{2} / 2\right)
$$

with $\mathrm{L}_{k}$ the Laguerre polinomials and $a$ and $c_{k}$ variational parameters. Up to 10 polinomials have been included in the present calculations.

The resulting ground state energies in units $\lambda_{1} / b$ for different values of $m$ are shown in Table 1 (the second column). Considered as a function of $m$ the ground state energy $E(m)$ has a sharp minimum at $m=1$, which can be determined exactly from the known intercept of the BFKL pomeron. With the growth of $m$ the energy first goes down very steeply at small values of $m-1$ but then flattens and beginning from $m-1 \sim 0.1$ diminishes very slowly with $m$. For $m$ greater than 2 the obtained values for the ground state energy do not practically depend on $m$ up to $m=6$, changing from 1.3 to 1.1. Also the optimal values of the parameter $a$ are shown in Table 1 (the third column). These characterize the typical values of $q^{2}$ for the gluons. For $m=1$ a goes to zero: as mentioned small values of $q^{2}$ are essential in this case, which makes it completely unphysical. However with the growth of $m$ the average values of $q^{2}$ rapidly grow to become considerably greater than 1 for $m^{2}$ greater than 1.1. Then the picture becomes self-consistent, restricted to the region of high momenta. In the last column of Table 1 the values of the intercept are shown for QCD with three flavours $\left(\lambda_{1} / b=4 / 3\right)$. For all values of $m$, except the ones quite close to 1 , the intercept results considerably smaller than for the BFKL pomeron. Its typical values are of the order of 0.15 . It is remarkarble that such values are close to the ones usually taken for the pomeron intercept in the semiphenomenological description of soft strong phenomena in the framework of the supercritical pomeron model [ 4 ].

\section{Acknowledgments}

The author is deeply grateful to E. M. Levin, L. N. Lipatov, C. Pajares and N. Armesto for fruitful and constructive discussions. He also expresses his gratitude to the General Direction of the Scientific and Technical Investigation (DGICYT) of Spain for financial support. 


\section{$7 \quad$ References}

1. E. A. Kuraev, L. N. Lipatov and V.S. Fadin, Sov. Phys. JETP 45 (1977) 199;

Ya. Ya. Balitzky and L. N. Lipatov, Sov. J. Nucl. Phys. 28 (1978) 822.

2. E. M. Levin and M. G. Ryskin, Phys. Rep. 189 (1990) 267;

N. N. Nikolaev and B. G. Zakharov, Phys. Lett. B327 (1994) 157;

J. Kwiecinski, A. D. Martin, P. J. Sutton and K. Golec-Biernat, preprint DTP/94/08.

3. L. N. Lipatov in "Perturbative Quantum Chromodynamics", Ed. A. H. Mueller, World Scientific 1989.

4. A. Capella, U. P. Sukhatme, C.-I. Tan and J. Tran Thanh Van, Phys. Rep. 236 (1994) 225

A. B. Kaidalov and K. A. Ter-Martirosyan, Phys. Lett. B117 (1982) 247. 


\section{Table}

\begin{tabular}{|r|r|r|r|}
\hline$m^{2} / \Lambda^{2}$ & $E$ & $a=<q^{2}>/ \Lambda^{2}$ & $\Delta$ \\
\hline 1.0 & -2.04 & 0. & 2.72 \\
\hline 1.01 & -0.518 & 0.50 & 0.690 \\
\hline 1.05 & -0.309 & 1.50 & 0.412 \\
\hline 1.1 & -0.238 & 2.56 & 0.317 \\
\hline 1.5 & -0.145 & 11.3 & 0.193 \\
\hline 2.0 & -0.129 & 24.0 & 0.172 \\
\hline 3.0 & -0.119 & 54.4 & 0.159 \\
\hline 4.0 & -0.114 & 90.0 & 0.152 \\
\hline 6.0 & -0.109 & 172.0 & 0.145 \\
\hline
\end{tabular}

\section{Table captions}

The first column gives values of the gluon mass squared in units $\Lambda^{2}$. In the second column ground state pomeron energies are presented in units $\lambda_{1} / b$. The third column gives the optimal values of the variational parameter $a$ which correspond to average gluon momentum squared in units $\Lambda^{2}$. The last column gives the pomeron intercept (minus one) for the QCD with three flavours $(b=9 / 4)$. 